\title{
Pulmonary crystalline silica exposure: the adverse effects
}

\section{Editorial}

Inhalation of free crystalline silica (size between 3-5microns) can contribute to the diseases include silicosis, pulmonary tuberculosis (TB), industrial bronchitis with air flow limitation, and several extra pulmonary diseases, such as scleroderma, rheumatoid arthritis, renal glomerular injury (glomerulonephritis), and other autoimmune diseases. The three important crystalline forms of silica are quartz, tridymite, and cristobalite, called "free silica". The "free silica" distinguishes them from the silicates, minerals containing silica bound to one or more metallic cations. Although people who do not work in a dusty trade, they may come into contact with the respirable crystalline silica and can develop pulmonary silicosis. Exposure to silica of a respirable size occurs in mining, drilling, tunneling, and quarrying operations. Potential health effects from short period (several months or years) of exposure to air-borne silica or residence in locations where prevailing winds carry silica particles from natural or industrial sites may be raised to public concern. These forms of silicosis are called "acute silicosis"and "accelerated silicosis". Nevertheless, chronic simple silicosis, the most common form has been described after environmental exposures to silica in regions where dust storms are common and soil silica content is high. Mild mixed dust pneumoconiosis without pulmonary silicotic nodules has also been described in agricultural workers. It is common for silicosis to be diagnosed after a worker has left the silica-exposed industry, and for it to continue to progress slowly over many decades of life after exposure has ended. Workers in many industries and occupations are at risk for silica exposure are construction, particularly, bridge, and elevated highway, wrecking and demolition, concrete work, stone cutting, milling stone, agriculture, foundry, ceramics, clay, pottery, vitreous enameling of China plumbing fixtures, glass manufacturing, manufacturing of concrete products and brick, manufacturing of soaps and detergents, railroads, and shipyards. A study of South African gold miners after they had left the mining industry showed a $25 \%$ cumulative risk of silicosis after 28years of mining at a $0.33 \mathrm{mg} /$ $\mathrm{m} 3$ silica exposure level. Study of Hong Kong granite quarries demonstrated that cumulative silica exposure between 1 and $5 \mathrm{mg} /$ $\mathrm{m} 3$ per year contribute to radiologic silicosis in $32 \%$ of men 50year and older. In the early stages, macroscopically, one can identify small dark (in association with anthracosis) or pale nodules, located predominantly in the upper lobes of lungs. In the later stages, of disease, the fibrous nodules enlarge and merge, resulting in large areas of pulmonary fibrosis. These nodules, namely "silica nodules" can present central ischemic necrosis. Microscopically, silica nodules consist of concentric laminated collagen fibers and tend to become confluent, compressing adjacent alveoli. With progression, perivascular and peribronchiolar collagen deposits will produce pulmonary hypertension.

Acute silicosis is recognized by rapid clinical manifestations. Chest roentgen graphic findings show diffuse alveolar bibasilar opacities representing fluid-filled alveoli. High-resolution (thin-
Volume 3 Issue I - 2016

\author{
Attapon Cheepsattayakorn, ${ }^{1,2,3}$ Ruangrong \\ Cheepsattayakorn ${ }^{4}$ \\ 'Editor-in-Chief, Journal of Lung Pulmonary and Respiratory \\ Research, USA \\ ${ }^{2}$ Oth Zonal Tuberculosis and Chest Disease Center,Thailand \\ ${ }^{3} 5$ th Office of Disease Prevention and Control, Department of \\ Disease Control, Ministry of Public Health, Thailand \\ ${ }^{4}$ Department of Pathology, Chiang Mai University, Thailand
}

Correspondence: Attapon Cheepsattayakorn, I0th Zonal Tuberculosis and Chest Disease Center, I 43 Sridornchai Road Changklan Muang Chiang Mai 50100 Thailand,Tel 6653 140767, 6653 276364, Fax 6653 140773, 6653 273590, Email Attapon1958@gmail.com, attaponche@yahoo.com

Received: January 16, 2016 | Published: January 18, 2016

section), spiral computed tomographic scanning is the best diagnostic method to demonstrate areas of ground-glass density that consists of reticular infiltration and areas of patchy increased attenuation and in homogeneity. The adjunctive diagnostic tests are sputum culture and cytology, PET scanning, pulmonary function tests, measures of gas exchange during exercise (pulse oximetry), measures of serum antinuclear antibodies and rheumatoid factor, and bronchoscopy. Silicosis-related TB which is related to the underlying prevalence of prior latent tuberculous infection has long been recognized. The risk of new exposure to TB of the population at risk for silicosis is also reported. Patients with chronic silicosis have a 3 -fold increase in incidence of TB, compared with a similarly aged, silica-exposed group without silicosis. In acute and accelerated silicosis, the incidence of TB and non-tuberculous mycobacterial (NTM) diseases is highest, whereas, in chronic silicosis, the incidence of active TB increases in direct proportion with the increase in the profusion of silicotic nodules, and with the highest category of perfusion reaches levels of risk comparable with those reported for HIV-infected persons. Pleural effusion has been reported in a 77-year-old man with almost 50 years' history of occupational silica exposure. Persons with periods of exposure to silica longer than 10years, even without disease, should be performed the tuberculin skin tests. If the result is negative, the test must be performed again. If the negative result persists, the test should be performed annually. If the result is positive (in duration is $10 \mathrm{~mm}$. or more), chemoprophylaxis for TB is indicated. The recommended management of silicosis-related TB is the same as the management of TB without silicosis. The current recommended chemoprophylaxis is isoniazid $300 \mathrm{mg}$ daily for one year, but some investigators recommend isoniazid $300 \mathrm{mg}$ daily for 6 months. Chemoprophylaxis 
for NTM infection in this situation has not been studied. A study revealed that rifampicin plus ethambutol for 9months is effective for silicosis-related Mycobacterium kansaii infection. Patients with silicosis are more likely to die with pulmonary mycosis, particularly, aspergillosis than are those without pneumoconiosis or those with more common pneumoconiosis. Treatment for pulmonary silicosis includes removal from further exposure which is the best measure, empiric use of bronchodilators and inhaled corticosteroids for airway obstruction, oral corticosteroids, whole lung larvage (sometimes), and lung transplantation (rarely).

\section{Acknowledgements}

None.

\section{Conflict of interest}

The author declares no conflict of interest. 